HD-THEP-96-01

\title{
The Ward Identity from the Background Field Dependence of the Effective Action
}

\author{
F. Freire' and C. Wetterich" \\ Institut für Theoretische Physik, Universität Heidelberg, \\ Philosophenweg 16, D-69120 Heidelberg, Germany
}

\begin{abstract}
The dependence of the effective action for gauge theories on the background field obeys an exact identity. We argue that for Abelian theories the Ward identity follows from the more general background field identity. This observation is particularly relevant for the anomalous Ward identity valid for gauge theories with an effective infrared cutoff as used for flow equations.
\end{abstract}

\footnotetext{
${ }^{1}$ E-mail: F.Freire@ThPhys.Uni-Heidelberg.DE

${ }^{2}$ E-mail: C.Wetterich@ThPhys.Uni-Heidelberg.DE
} 
The effective average action $\Gamma_{k}$ is a useful concept for the investigation of infrared problems. In field theory it corresponds to the quantum effective action with an effective infrared cutoff $\sim k$ for the fluctuations. In statistical physics it is the coarse grained free energy with coarse graining length scale $\sim k^{-1}$. Exact non-perturbative flow equations describe the dependence of $\Gamma_{k}$ on $k$ [1]. They are related to the Wilsonian approach to the renormalization group equations [2]. Since non-Abelian gauge theories are plagued in perturbation theory by severe infrared problems the use of the effective average action seems particularly promising here. Writing down exact flow equations for gauge theories poses no additional difficulty. The problem of correct implementation of gauge symmetry arises rather on the level of their solutions. In fact, models with local gauge symmetry correspond to particular trajectories in the space of general solutions of the flow equations. It is crucial to find appropriate identities for $\Gamma_{k}$ which enforce a restriction to "gaugeinvariant solutions" and guarantee gauge invariance for $k \rightarrow 0$.

So far, two different lines of research have been followed in this respect. The first one [3] [4] works in the background field formalism where $\Gamma_{k}[A, \bar{A}]$ depends on the classical gauge field $A_{\mu}$ (conjugate to the source) and the background gauge field $\bar{A}_{\mu}$ which enters through the gauge fixing and the infrared cutoff. By construction $\Gamma_{k}[A, \bar{A}]$ is invariant under simultaneous gauge transformations of $A_{\mu}$ and $\bar{A}_{\mu}$. This combined gauge invariance is, however, not sufficient to guarantee that the solution lies on a trajectory appropriate for a gauge-invariant theory. An additional exact identity for the background field dependence $\delta \Gamma_{k} / \delta \bar{A}_{\mu}$ was derived [4] and it is believed that this identity guarantees full gauge invariance of the theory. The second approach [5] 6] centers on the Ward-Takahashi or Slavnov-Taylor identities. These identities receive anomalous contributions [6] from the presence of the infrared cutoff term $\sim R_{k}$ which vanish only for $k \rightarrow 0$. Obviously, the same type of identities can also be derived within the background field formalism. One would suspect that the background field identity and the Ward identity are not unrelated since both reflect the content of gauge symmetry. Their exact relation has, however, not been revealed in the past. 
In this letter we indicate how the Ward identity can be derived from the background field dependence of the effective action for Abelian gauge theories. The model we use is scalar electrodynamics (SQED) in arbitrary dimension $d$. The classical action consists of the usual SQED action plus a gauge-fixing term and a quadratic term implementing an infrared cutoff. For the complex scalar field $\chi(x)$ the infrared cutoff reads [ [⿴囗十

$$
\Delta_{k}^{(S)} S=\int d^{d} x \chi^{*}(x) R_{k}(\mathcal{D}[\bar{A}]) \chi(x)
$$

Here $R_{k}$ is a function that cuts off the modes with momentum smaller than $k$ with $R_{k}(0) \sim k^{2}$. More precisely, the cutoff distinguishes between eigenvalues of the covariant Laplacian, $\mathcal{D}[\bar{A}]=-\left(\partial_{\mu}+i g \bar{A}_{\mu}(x)\right)\left(\partial^{\mu}+i g \bar{A}^{\mu}(x)\right)$, where $\bar{A}_{\mu}(x)$ is the background gauge field. The complete set of quadratic cutoff terms includes a term for the gauge sector which can be chosen to be gauge invariant in Abelian gauge theories. If this is the case it does not affect the Ward identity or the background field identity and does not need to be specified for our discussion.

We choose the background gauge fixing

$$
\Gamma_{\mathrm{gf}}[A, \bar{A}]=\frac{1}{2 \alpha} \int d^{d} x\left(\partial_{\mu}\left[A^{\mu}(x)-\bar{A}^{\mu}(x)\right]\right)^{2},
$$

and consider the effective action $\Gamma_{k}[\varphi, A, \bar{A}]$ with $\varphi$ the classical scalar field related to $\chi$. For this gauge fixing it has been shown that the background field dependence of $\Gamma_{k}$ is governed by the identity $[4$

$$
\frac{\delta \Gamma_{k}}{\delta \bar{A}_{\mu}}=\operatorname{Tr}\left\{\frac{\delta \mathcal{R}_{k}^{(\varphi)}}{\delta \bar{A}_{\mu}}\left(\Gamma_{k}^{(2)}+\mathcal{R}_{k}\right)^{-1}\right\}+\frac{1}{\alpha} \partial^{\mu} \partial_{\nu}\left(A^{\nu}-\bar{A}^{\nu}\right)
$$

where the arguments of the functionals have been omitted. In Eq. (3) Tr stands for integration over the configuration or momentum space, e.g. $\operatorname{Tr}=\int \frac{d^{d} p}{(2 \pi)^{d}}$ whilst $\left(\Gamma_{k}^{(2)}+\mathcal{R}_{k}\right)^{-1}$ denotes the inverse of the two-point connected Green function for the complex scalar field in the presence of the infrared cutoff. In momentum space where $\left(\Gamma_{k}^{(2)}+\mathcal{R}_{k}\right)_{\varphi\left(p^{\prime}\right) \varphi(p)}=$ $\frac{\delta}{\delta \varphi\left(p^{\prime}\right)} \frac{\delta}{\delta \varphi^{*}(p)}\left(\Gamma_{k}+\Delta_{k} S\right)$, we have 


$$
\left(\Gamma_{k}^{(2)}+\mathcal{R}_{k}\right)_{\varphi\left(p^{\prime}\right) \varphi(p)}^{-1}=\left\langle\chi^{*}\left(p^{\prime}\right) \chi(p)\right\rangle_{c}
$$

By subtracting the gauge-fixing term

$$
\Gamma_{k}^{\prime}[\varphi, A, \bar{A}]=\Gamma_{k}[\varphi, A, \bar{A}]-\Gamma_{\mathrm{gf}}[A, \bar{A}]
$$

Eq. (3) becomes

$$
\frac{\delta \Gamma_{k}^{\prime}}{\delta \bar{A}_{\mu}}=\operatorname{Tr}\left\{\frac{\delta \mathcal{R}_{k}^{(\varphi)}}{\delta \bar{A}_{\mu}}\left(\Gamma_{k}^{(2)}+\mathcal{R}_{k}\right)^{-1}\right\}
$$

The Ward identity for SQED in the absence of cutoff functions is well-known. In the presence of the infrared cutoff term $\Delta_{k}^{(S)} S$ it receives an anomalous contribution [6]. For our choice of the gauge-fixing term the anomalous Ward identity reads

$$
\begin{aligned}
& \frac{1}{g} q_{\mu} \frac{\delta \Gamma_{k}^{\prime}}{\delta A_{\mu}(q)}+\int \frac{d^{d} p}{(2 \pi)^{d}}\left\{\frac{\delta \Gamma_{k}^{\prime}}{\delta \varphi(p)} \varphi(p-q)-\frac{\delta \Gamma_{k}^{\prime}}{\delta \varphi^{*}(p)} \varphi^{*}(p+q)\right\}= \\
& \int \frac{d^{d} p}{(2 \pi)^{d}} \frac{\mathrm{d}^{d} p^{\prime}}{(2 \pi)^{d}}\left\{\mathcal{R}_{k}^{(\varphi)}\left(p+q, p^{\prime}\right)-\mathcal{R}_{k}^{(\varphi)}\left(p, p^{\prime}-q\right)\right\}\left(\Gamma_{k}^{(2)}+\mathcal{R}_{k}\right)_{\varphi\left(p^{\prime}\right) \varphi(p)}^{-1} .
\end{aligned}
$$

Note that $\mathcal{R}_{k}^{(\varphi)}\left(p, p^{\prime}\right)$ is not diagonal in momentum space $\left(\partial_{\mu}\right.$ and $\bar{A}_{\mu}(\mathrm{x})$ do not commute) and depends on $\bar{A}_{\mu}$, Eq. (13). The right-hand side of Eq. (7) vanishes for $k=0\left(\mathcal{R}_{k}=0\right)$ and the identity reduces to the standard homogeneous linear relation between one-particle irreducible Green functions. In this case the identity is known to encode the transversality of the effects from fluctuations in the propagator of the gauge field and it can be seen as a constraint guaranteeing the absence of non-physical (in this instance longitudinal) degrees of freedom. In the presence of the infrared cutoff the Ward identity is a generalisation of this constraint. Truncated solutions to the exact flow equation should satisfy them at least approximately such that the homogeneous Ward identity is recovered for $k=0$.

We now want to show that the Ward identity, Eq. (7), follows from the background field identity, Eq. (3). The invariance of the background field effective action under a simultaneous gauge transformation of $\varphi, A$, and $\bar{A}$ results in the identity

$$
\frac{1}{g} q_{\mu} \frac{\delta \Gamma_{k}^{\prime}}{\delta A_{\mu}(q)}+\frac{1}{g} q_{\mu} \frac{\delta \Gamma_{k}^{\prime}}{\delta \bar{A}_{\mu}(q)}+\int \frac{d^{d} p}{(2 \pi)^{d}}\left\{\frac{\delta \Gamma_{k}^{\prime}}{\delta \varphi(p)} \varphi(p-q)-\frac{\delta \Gamma_{k}^{\prime}}{\delta \varphi^{*}(p)} \varphi^{*}(p+q)\right\}=0 .
$$


From Eq. (8) the proof that Eq. (4) is contained in Eq. (6) follows immediately if we show that the following equality holds

$$
\begin{aligned}
& \frac{1}{g} q_{\mu} \int \frac{d^{d} p}{(2 \pi)^{d}} \frac{d^{d} p^{\prime}}{(2 \pi)^{d}} \frac{\delta \mathcal{R}_{k}^{(\varphi)}\left(p, p^{\prime}\right)}{\delta \bar{A}_{\mu}(q)}\left(\Gamma_{k}^{(2)}+\mathcal{R}_{k}\right)_{\varphi\left(p^{\prime}\right) \varphi(p)}^{-1}= \\
& \quad-\int \frac{d^{d} p}{(2 \pi)^{d}} \frac{\mathrm{d}^{d} p^{\prime}}{(2 \pi)^{d}}\left\{\mathcal{R}_{k}^{(\varphi)}\left(p+q, p^{\prime}\right)-\mathcal{R}_{k}^{(\varphi)}\left(p, p^{\prime}-q\right)\right\}\left(\Gamma_{k}^{(2)}+\mathcal{R}_{k}\right)_{\varphi\left(p^{\prime}\right) \varphi(p)}^{-1}
\end{aligned}
$$

(Our computation in momentum space avoids problems due to operator ordering in $\frac{\delta \mathcal{R}_{k}[\bar{A}]}{\delta \bar{A}_{\mu}}$.) We need to evaluate $\mathcal{R}_{k}^{(\varphi)}\left(p, p^{\prime}\right)$ which corresponds to $R_{k}(\mathcal{D}[\bar{A}])$ in a Fourier basis $\left(\varphi(p)=\int d^{d} x \varphi(x) \exp (i p x)\right)$

$$
\int d^{d} x \varphi^{*}(x) R_{k}(\mathcal{D}[\bar{A}]) \varphi(x)=\int \frac{d^{d} p}{(2 \pi)^{d}} \frac{d^{d} p^{\prime}}{(2 \pi)^{d}} \mathcal{R}_{k}^{(\varphi)}\left(p, p^{\prime}\right) \varphi^{*}\left(p^{\prime}\right) \varphi(p) .
$$

For this purpose we expand $R_{k}$ in a power series of its argument

$$
R_{k}(\mathcal{D}[\bar{A}])=\sum_{n=0}^{\infty} \frac{1}{n !} R_{k}^{(n)}(0)(\mathcal{D}[\bar{A}])^{n},
$$

where $R_{k}^{(n)}(0)=\left.\frac{\mathrm{d}^{n} R_{k}(x)}{\mathrm{d} x^{n}}\right|_{x=0}$. Inserting

$$
\begin{aligned}
\mathcal{D}\left(p, p^{\prime}\right)=p^{2} \bar{\delta}\left(p-p^{\prime}\right)-g \int \frac{d^{d} Q}{(2 \pi)^{d}} \bar{A}^{\mu}(Q)\left(p+p^{\prime}\right)_{\mu} \bar{\delta}\left(p+Q-p^{\prime}\right) \\
+g^{2} \int \frac{d^{d} Q}{(2 \pi)^{d}} \frac{d^{d} Q^{\prime}}{(2 \pi)^{d}} \bar{A}^{\mu}(Q) \bar{A}_{\mu}\left(Q^{\prime}\right) \bar{\delta}\left(p+Q+Q^{\prime}-p^{\prime}\right)
\end{aligned}
$$

and comparing powers of $\bar{A}_{\mu}$ one arrives after a straightforward but lengthy manipulation at

$$
\begin{gathered}
\mathcal{R}_{k}^{(\varphi)}\left(p, p^{\prime}\right)=R_{k}\left(p^{2}\right) \bar{\delta}\left(p-p^{\prime}\right)-g \int \frac{d^{d} Q}{(2 \pi)^{d}} \bar{A}^{\mu}(Q)\left(p+p^{\prime}\right)_{\mu} \frac{R_{k}\left(p^{2}\right)-R_{k}\left(p^{\prime 2}\right)}{p^{2}-p^{\prime 2}} \bar{\delta}\left(p+Q-p^{\prime}\right) \\
+g^{2} \int \frac{d^{d} Q}{(2 \pi)^{d}} \frac{d^{d} Q^{\prime}}{(2 \pi)^{d}} \bar{A}^{\mu}(Q) \bar{A}^{\nu}\left(Q^{\prime}\right) \bar{\delta}\left(p+Q+Q^{\prime}-p^{\prime}\right)\left\{\delta_{\mu \nu} \frac{R_{k}\left(p^{2}\right)-R_{k}\left(p^{\prime 2}\right)}{p^{2}-p^{\prime 2}}\right. \\
-\frac{(2 p+Q)_{\mu}\left(2 p^{\prime}-Q^{\prime}\right)_{\nu}}{(p+Q)^{2}-p^{2}}\left[\frac{R_{k}\left(p^{2}\right)-\frac{p^{2}}{p^{\prime 2}} R_{k}\left(p^{\prime 2}\right)}{p^{2}-p^{\prime 2}}\right. \\
\left.\left.-\frac{R_{k}\left(\left(p^{\prime}-Q^{\prime}\right)^{2}\right)-\frac{\left(p^{\prime}-Q^{\prime}\right)^{2}}{p^{\prime 2}} R_{k}\left(p^{\prime 2}\right)}{\left(p^{\prime}-Q^{\prime}\right)^{2}-p^{\prime 2}}\right]\right\}+\mathcal{O}\left(g^{3}\right),
\end{gathered}
$$


Here $R_{k}\left(p^{2}\right)$ equals $R_{k}\left(-\partial^{2}\right)$ in a momentum space representation and $\bar{\delta}(p)=(2 \pi)^{d} \delta(p)$. We observe that $\mathcal{R}_{k}^{(\varphi)}$ becomes diagonal only for $\bar{A}_{\mu}(x)=$ const.

A direct computation of $\frac{\delta \mathcal{R}_{k}^{(\varphi)}\left(p, p^{\prime}\right)}{\delta \bar{A}_{\mu}(q)}$ in order $g^{2}$ can be carried out using Eq. (13) and one wants to establish

$$
\frac{1}{g} q_{\mu} \frac{\delta \mathcal{R}_{k}^{(\varphi)}\left(p, p^{\prime}\right)}{\delta \bar{A}_{\mu}(q)}=\mathcal{R}_{k}^{(\varphi)}\left(p, p^{\prime}-q\right)-\mathcal{R}_{k}^{(\varphi)}\left(p+q, p^{\prime}\right) .
$$

It is easy to verify this in lowest order. We have in addition checked the first nontrivial order in $\bar{A}_{\mu}$ which is $\mathcal{O}(g)$. This shows that Eq. (9) holds at least in this order, and we conclude that the Ward identity, Eq. (7), is contained in the identity for the background field dependence of the effective action $\Gamma_{k}$, Eq. (3).

We have no reasons to doubt that Eq. (14) is an exact identity valid in all orders in $g$. It holds trivially if only constant background fields are considered whereas a proof for general $\bar{A}_{\mu}(x)$ needs more refined methods. We also expect that the background field identity implies the Ward identity for all Abelian gauge theories with arbitrary matter fields. This clarifies at least for the Abelian case the relation between the two mentioned approaches to the use of exact flow equations for gauge theories. In particular, the standard covariant gauge fixing is a special case of Eq. (2) for $\bar{A}_{\mu}(x)=0$. The background field identity, Eq. (6), can also be used at $\bar{A}_{\mu}(x)=0$. For general $\bar{A}_{\mu}(x)$ Eq. (6) is stronger than the Ward identity since the latter is equivalent to its divergence, or, in momentum space, to a contraction with $q^{\mu}$. For practical applications one may isolate the gauge-invariant kernel $\bar{\Gamma}_{k}[\varphi, A]=\Gamma_{k}[\varphi, A, \bar{A}=A]$ and expand the remaining background field dependent part, $\hat{\Gamma}_{k}^{\text {gauge }}[\varphi, A, \bar{A}]=\Gamma_{k}^{\prime}[\varphi, A, \bar{A}]-\bar{\Gamma}_{k}[\varphi, A]$, in powers of $A(x)-\bar{A}(x)$

$$
\begin{aligned}
\hat{\Gamma}_{k}^{\text {gauge }}[\varphi, A, \bar{A}]=\int d^{d} x\left\{H_{1}^{\mu}[\varphi, \bar{A}]\left(A_{\mu}(x)-\bar{A}_{\mu}(x)\right)\right. \\
\left.\quad+\frac{1}{2} H_{2}^{\mu \nu}[\varphi, \bar{A}]\left(A_{\mu}(x)-\bar{A}_{\mu}(x)\right)\left(A_{\nu}(x)-\bar{A}_{\nu}(x)\right)+\ldots\right\} .
\end{aligned}
$$

Here $H_{i}$ are gauge-invariant Lorentz-tensors which may depend on $\varphi(x)$ and $\bar{A}_{\mu}(x)$ but not on $A_{\mu}(x)$. For any given $\bar{\Gamma}_{k}[\varphi, A]$ all $H_{i}$ can be computed from appropriate functional 
derivatives of Eq. (6) with $\Gamma_{k}^{\prime}$ replaced by $\hat{\Gamma}_{k}^{\text {gauge }}$. For example, a photon mass term for $\varphi=0$ is contained in $H_{2}^{\mu \nu}=m_{A}^{2} \delta^{\mu \nu}+\ldots$ The correction from $\hat{\Gamma}_{k}^{\text {gauge }}$ to the photon two-point function reads

$$
\Delta G^{\mu \nu}(q) \bar{\delta}\left(q-q^{\prime}\right)=-\left[\frac{\delta^{2} \hat{\Gamma}_{k}^{\text {gauge }}}{\delta \bar{A}_{\mu}(q) \delta \bar{A}_{\nu}\left(-q^{\prime}\right)}+\frac{\delta^{2} \hat{\Gamma}_{k}^{\text {gauge }}}{\delta A_{\mu}(q) \delta \bar{A}_{\nu}\left(-q^{\prime}\right)}+\frac{\delta^{2} \hat{\Gamma}_{k}^{\text {gauge }}}{\delta \bar{A}_{\mu}(q) \delta A_{\nu}\left(-q^{\prime}\right)}\right]_{0}
$$

where $[\ldots]_{0}$ means evaluated at $A=\bar{A}=\varphi=0$. The photon mass term is extracted for $q=0\left(\Delta G^{\mu \nu}(0)=m_{A}^{2} \delta^{\mu \nu}\right)$ and one finds from Eq. (6) that it vanishes for $k \rightarrow 0$ as $m_{A}^{2} \sim g^{2} k^{2}$. We conclude that $\hat{\Gamma}_{k}^{\text {gauge }}$ is completely fixed in terms of $\bar{\Gamma}_{k}$ by the background field identity Eq. (6) — at least as long as the part $\hat{\Gamma}_{k}^{\text {gauge }(2)}$ in $\Gamma_{k}^{(2)}$ on the right-hand side can be treated iteratively.

A background field identity has also been derived for non-Abelian gauge theories [3] and up to now has been little exploited. It would be very interesting to understand its relation with the anomalous Slavnov-Taylor identities [6]. In view of the findings of this letter for the Abelian case one may suspect that the non-Abelian background field identity contains much relevant information beyond the Slavnov-Taylor identity. 


\section{References}

[1] C. Wetterich, Z. Phys. C57 (1993) 451; C60 (1993) 461;

Phys. Lett. B301 (1993) 90;

M. Bonini, M. D’Attanasio and G. Marchesini, Nucl. Phys. B409 (1993) 441.

[2] F. Wegner and A. Houghton, Phys. Rev. A8 (1973) 401;

K. G. Wilson and I. G. Kogut, Phys. Rep. 12 (1974) 75;

S. Weinberg, Critical Phenomena for Field Theorists, Erice Subnucl. Phys. (1976) 1;

J. Polchinski, Nucl. Phys. B231 (1984) 269.

[3] M. Reuter and C. Wetterich, Nucl. Phys. B391 (1993) 147;

B408 (1993) 91; B417 (1994) 181; preprint HD-THEP-94-39.

[4] M. Reuter and C. Wetterich, Nucl. Phys. B427 (1994) 291.

[5] M. Bonini, M. D'Attanasio and G. Marchesini, Nucl. Phys. B418 (1994) 81;

B421 (1994) 429; B437 (1995) 163; Phys. Lett. B346 (1995) 87.

[6] U. Ellwanger, Phys. Lett. B335 (1994) 364;

U. Ellwanger, M. Hirsch and A. Weber, preprint LPTHE Orsay 95-39, to appear in Z. Phys. C 